\begin{tabular}{|c|c|}
\hline \multirow{3}{*}{ 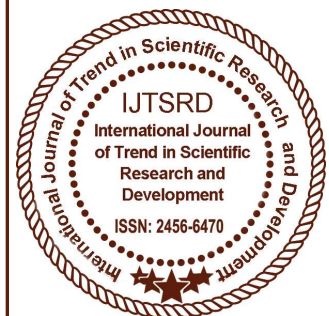 } & $\begin{array}{l}\text { International Journal of Trend in Scientific } \\
\text { Research and Development (IJTSRD) }\end{array}$ \\
\hline & International Open Access Journal \\
\hline & ISSN No: 2456 - 6470 | www.ijtsrd.com | Volume - 2 | Issue - 4 \\
\hline
\end{tabular}

\title{
A Survey on Feature Extraction Technique in Image Processing
}

\author{
Dr. A. Hema (MCA, M.Phil, Ph.D, PGDBI) ${ }^{1}$, R. Saravanakumar (MSc, M.Phil) ${ }^{2}$ \\ ${ }^{1}$ Associate Professor and Head, ${ }^{2}$ Ph.D, Research Scholar
}

Department of Computer Applications, Kongunadu Arts and Science College, Coimbatore, Tamil Nadu, India

\begin{abstract}
Image processing is a method to convert an image into digital form and perform some operations on it. It is a type of signal dispensation in which input is image, video frame or photograph and output may be image or characteristics associated with that image. Image processing basically include the nthree steps as importing the image with optical scanner or by digital photography. Analyzing and manipulating the image which includes data compression and image enhancement and spotting pattern that are not to human eyes like satellite photographs. Output is the last stage in which result can be altered and report is based on image analysis. In recent trends, image processing domain plays a virtual part of real time application in modern world. Feature extraction starts from an initial set of measured data and builds derived values (features) intended to be informative and nonredundant, facilitating the subsequent learning and generalization steps, some cases leading to better human interpretations.
\end{abstract}

Keywords: Image Processing and Feature Extraction.

\section{Introduction}

Image processing involves processing or altering an existing image in a desired manner and also helps in obtaining the image in the readable format. Benefits of Image processing is faster and cost effective, image sharpening and restoration-to create a better image, which can be retrieval easily from the database.Feature extraction is the most important step in image processing. It helps in extracting the feature of an image as ideal as possible. Feature extraction techniques are applied to get feature that will be useful in classifying and recognizing the images.
These methods are classified as low-level feature extraction and high-level feature extraction. Lowlevel feature extractions are based on finding the points, lines, edges, etc while high level feature extraction methods use the low level feature to provide more significant information for further processing of image analysis. Feature plays a very important role in the area of image processing. Before getting features, various image processing techniques like binarization, thersholding, resizing, normalization etc. are applied on the sampled image. After that, feature extraction techniques are applied to get features that will be useful in recognition of images. Feature extraction techniques are helpful in various image processing applications. As features define the behaviour of an image, the show its place in terms of storage taken.

Feature extraction techniques and explaining in what scenario, which feature extraction technique, will be better.

\section{Literature Survey}

Rajkumar et al, [1] describes, the efficiency of feature extraction method enhance the further processing of an image to a great extent. These features can be used in image matching, pattern recognition and retrieval. Feature extraction technique is used to extract that feature by keeping as much as information as possible from the large set of data of image. Numerous methods are used to extract features like color, texture and shape as feature vector.

Dixa Saxena et al, [2] to obtain the most relevant information from the original data and represent the 
information in a lower dimensionality space. Constructing combinations of the variable to get reduced number of features while still describing the data sufficient accuracy.

Asghar Feizi, [3] the feature have high discriminative power and low dimensional feature space. In the process of feature fusion, the features keep independent and contain redundant and overlapping information as little as possible. The feature are resistant to thorny challenges of person ReIdentification including low resolution, occlusion, arbitrary poses, background cutter and variation in illumination.

Monika et al, [4] the objective of the features extraction is to capture important characteristics of region under investigation in the kidney images. The features of the region should be able to identity region uniquely. The present research has used statistical features to identify the region of interest. The concept of gray level co-occurrence matrix (GLCM) used to extract the features.

Elavarasan et al, [5] deals, the technique is used to extracts a subset of new features from the original feature set by means of some functional mapping by keeping as much information in the data as possible. The following methods are commonly used for the features extraction. The follwing methods are Principal Component Analysis (PCA) and Linear Discriminant Analysis (LDA). PCA method extracts a lower dimensional space by analyzing the covariance structure of multivariate statistical observations. LDA mainly projects the highdimensional data into lower dimensional space.

Shreya Narang et al, [6] explain, feature extraction is the main part of the speech recognition system. It is considered as the heart of the system. The work of this is to extract those features from the input speech (signal) that help the system in identifying the speaker. Feature extraction compresses the magnitude of the input signal (vector) without causing any harm to the power of speech signals.

Preeti et al, [7] says, the Images to extract patterns and derive knowledge from large collections of images which mainly deals with identification and extraction of unique features for a particular domain. Increasing amount of illicit image data transmitted via the internet has triggered the need to develop effective image mining system for digital forensics purpose.

Muhammad et al, [8] describes, feature extraction in HCR is a very important field of image processing and object recognition. Fundamental component of characters are called features. The basic task of feature extraction and selection is to find out group of the most effective features for classification that is compressing from high-dimensional feature space to low-dimensional feature space.

Wu Pin et al, [9] deals, new features represent raw data with linear or non-linear transformations, and the dimension of low-dimensional space are always the dimensions of sample data's features. Feature extraction can solve the issue which high-dimension data facing, using little but enough data to represent raw data to make recognizing and retrieving quickly. In this case, feature directly influences the whole system. Manual feature designing are a way that need people's wisdom and experience.

Gaurav et al, [10] presents the image data and finding the most appropriate feature extraction method, in order to utilize them in various application. Image is a different kind of data which includes a huge amount of information, such as color information, objects, edges, pixel definition, dimensions and others. The various key features and properties of image data by which the information from the image is extract and utilized for different application of face recognition, image retrieval and others.

Gaurav Kumar et al, [11] feature extraction done after the processing phase in character recognition system. The primary task of pattern recognition is to take an possible output and correctly assign it as one of the possible output classes. This process divided into two general stages: Feature selection and classification.

Ryszard et al, [14] the extraction task transforms rich content of images into various content features. Feature extraction is most critical because the particular features made available for discrimination directly. The end result of the extraction task is a set of features, commonly called a feature vector, which constitutes a representation of the image.

Sriram et al, [16] we propose a feature extraction technique based on two-dimensional (2-D) spectrotemporal AR models. The initial model is the 
temporal AR model based on frequency domain linear prediction. A robust feature extraction scheme must rely on the high energy regions in the spectrotemporal plane. In general, an autoregressive (AR) modeling approach represent high energy regions with good modeling accuracy. One-dimensional AR modeling of signals spectra is widely used for feature extraction of speech.

\section{Conclusion}

Image processing is a method to convert an image into digital form and perform some operations on it. It is a type of signal dispensation in which input is image, like video frame or photograph and output may be image or characteristics associated with that image.Many feature algorithms proposed by different researchers are discussed and the issues present in the existing algorithm were identified. Feature plays a very important role in the area of image processing. The various image processing techniques like binarization, thersholding, resizing, normalization etc. are applied on the sampled image. Feature extraction techniques are applied to get features that will be useful in recognition of images, and helpful in various image processing applications. Many auother's applied feature extraction techniquesin different applications. Accurate results are obtaining using those techniques.

\section{Bibiliography}

1) Rajkumar Goel, Vineet Kumar, Saurabh Srivastava, A. K. Sinha "A Review of Feature Extraction Techniques forImage Analysis" Noida Institute of Engineering \& Technology, Greater NoidaVol. 6, Special Issue 2, February 2017.

2) Dixa Saxena, S.K.Saritha, Ph.D, K. N. S. S. V. Prasad, "Survey Paper on Feature Extraction Methods in Text Categorization", International Journal of Computer Applications (0975 - 8887), Volume 166 - No.11, May 2017.

3) Asghar Feizi, "High-Level Feature Extraction for Classification and Person Re-Identification", IEEE SENSORS JOURNAL, VOL. 17, NO. 21, NOVEMBER 1, 2017.

4) Monika Pathak, Harsh Sadawarti, Sukhdev Singh "Features extraction and classification for detection of kidney stone region in ultrasound images", International Journal of Multidisciplinary Research and Development.Online ISSN: 2349-4182 Print
ISSN: 2349-5979, Impact Factor: RJIF 5.72. Volume 3; Issue 5; May 2016; Page No. 81-83.

5) N.Elavarasan1, Dr.K.Mani "A Survey on Feature Extraction Techniques", International Journal of Innovative Research in Computer and Communication Engineering, (An ISO 3297: 2007 Certified Organization)Vol. 3, Issue 1, January 2015.

6) Shreya Narang, Ms. Divya Gupta, "Speech Feature Extraction Techniques: A Review", International Journal of Computer Science and Mobile Computing, Vol.4 Issue.3, March- 2015, pg. 107-114.

7) Preeti Chouhan, Mukesh Tiwari "Image Retrieval Using Data Mining and Image Processing Techniques", INTERNATIONAL JOURNAL OF INNOVATIVE RESEARCH IN ELECTRICAL, ELECTRONICS, INSTRUMENTATION AND CONTROL ENGINEERING Vol. 3, Issue 12, December 2015.

8) Muhammad Arif Mohamad, Haswadi Hassan, Dewi Nasien, Habibollah Haron "A Review on Feature Extraction and Feature Selection for Handwritten Character Recognition, (IJACSA) International Journal of Advanced Computer Science and Applications, Vol. 6, No. 2, 2015.

9) $\mathrm{Wu}$ Pin, Yan Hongjie, Shang Weilie, Zhu Yonghua and Gao Honghao, "Research on Feature Extraction based on Deep Learning", International Journal of Hybrid Information Technology Vol.8, No.11 (2015), pp.113-120.

10) Gaurav Mandloi "A Survey on Feature Extraction Techniques for Color Images”, (IJCSIT) International Journal of Computer Science and Information Technologies, Vol. 5 (3) , 2014, 4615-4620.

11) Gaurav Kumar, Pradeep Kumar Bhatia, "A Detailed Review of Feature Extraction in Image Processing Systems", 2014 Fourth International Conference on Advanced Computing \& Communication Technologies.

12) Shikha Gupta, Jafreezal Jaafar, Wan Fatimah wan Ahmad and Arpit Bansal, "FEATURE EXTRACTION USING MFCC", Signal \& Image Processing : An International Journal (SIPIJ) Vol.4, No.4, August 2013. 
13) Rahimeh Rouhi, Mehran Amiri and Behzad Irannejad "A REVIEW ON FEATURE EXTRACTION TECHNIQUES IN FACE RECOGNITION", Signal \& Image Processing : An International Journal (SIPIJ) Vol.3, No.6, December 2012.

14) Ryszard S. Chora's "Image Feature Extraction Techniques and Their Applications for CBIR and Biometrics Systems",
JOURNAL OF BIOLOGY AND BIOMEDICAL ENGINEERING, Issue 1, Vol. 1, 2007.

15) H. Hassanpour and E. Hallajian, "Using Hidden Markov Models for Feature Extraction in Paper Currency Recognition".

16) Sriram Ganapathy, Samuel Thomas and Hynek Hermansky, "Feature Extraction Using 2-D Autoregressive Models For Speaker Recognition".

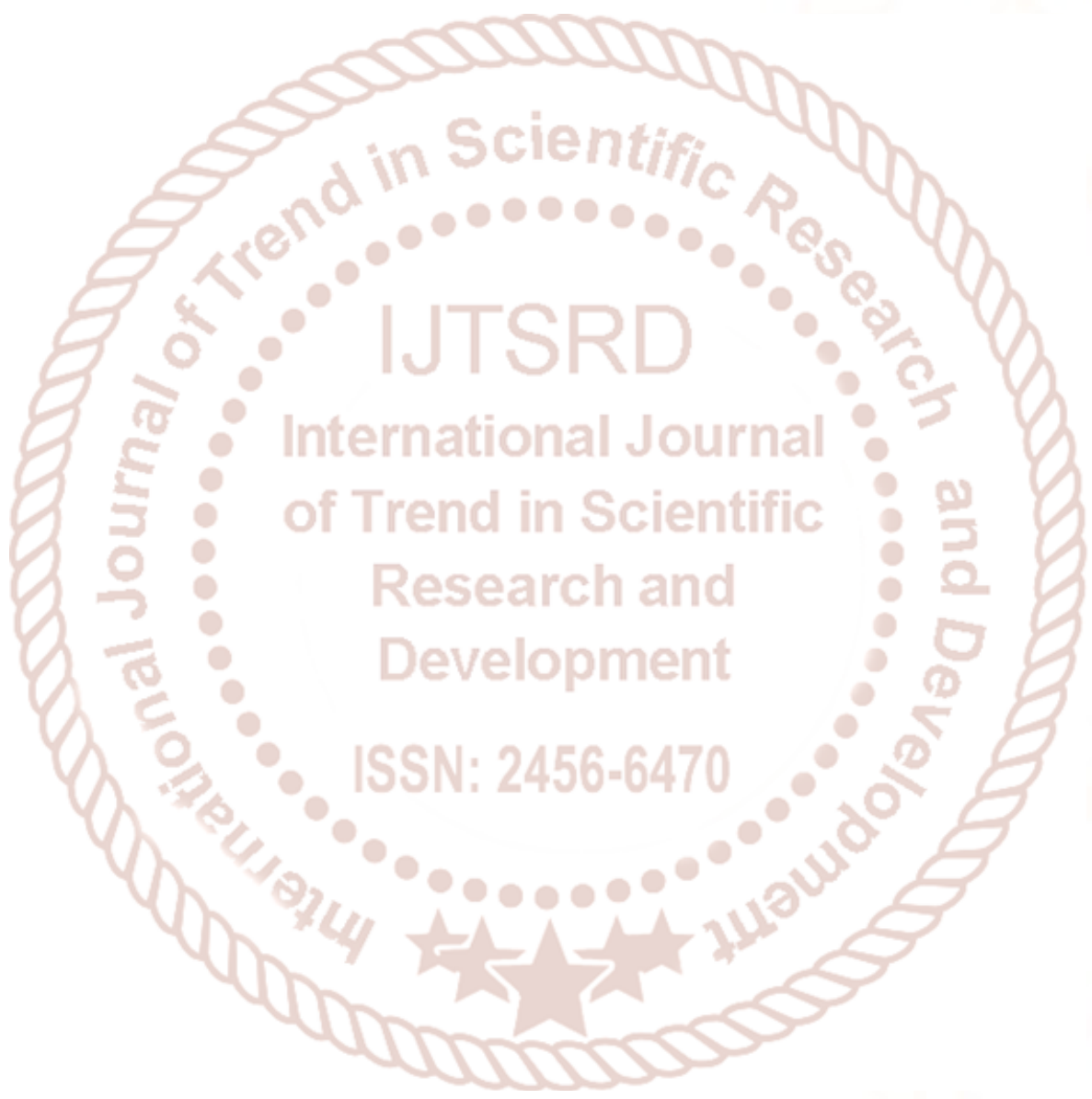

